\title{
Alfabetización transmedia y redes sociales: Estudio de caso de Instagram como herramienta docente en el aula universitaria
}

Transmedia literacy and social networks: Case study on Instagram as a teaching tool in the university classroom

\section{Alfabetização transmídia e redes sociais: estudo de caso do Instagram como ferramenta de ensino na sala de aula da universidade}

\author{
Nadia Alonso López \\ Profesora \\ Dpto. Comunicación Audiovisual, Documentación e $H^{a}$ del Arte \\ (Universitat Politècnica de València) \\ http://orcid.org/0000-0002-5220-2232 \\ España \\ Raúl Terol Bolinches \\ Profesor \\ Dpto. Comunicación Audiovisual, Documentación e $H^{a}$ del Arte \\ (Universitat Politècnica de València) \\ http://orcid.org/0000-0002-0915-7288 \\ España
}

Fecha de recepción: 20 de noviembre de 2019

Fecha de revisión: 9 de febrero de 2020

Fecha de aceptación: 5 de marzo de 2020

Fecha de publicación: 1 de julio de 2020

Para citar este artículo: Alonso López, N. y Terol Bolinches, R. (2020). Alfabetización transmedia y redes sociales: Estudio de caso de Instagram como herramienta docente en el aula universitaria, Icono 14, 18 (2), 138-161. doi: 10.7195/ri14.v18i2.1518 


\section{Resumen}

En el actual entorno mediático, los avances tecnológicos han dado lugar a nuevas formas de comunicación basadas en Internet, los dispositivos inteligentes y las redes sociales. En este contexto, las universidades reciben estudiantes pertenecientes a la llamada Generación $Z$, jóvenes que no conocen un mundo sin Internet, a quienes su teléfono inteligente acompaña constantemente y que emplean las redes sociales como principal herramienta de comunicación, fundamentalmente Instagram. Puesto que esta generación se basa en prácticas relacionadas con la tecnología y el desarrollo de competencias transmedia (Scolari, 2018), éstas pueden ser aprovechadas en el aula universitaria para facilitar la docencia integrándolas en los procesos de enseñanza-aprendizaje.

En la presente investigación, se estudia la implementación de las competencias transmedia en la enseñanza universitaria, concretamente con el caso de un canal de Instagram creado en el curso 2019-20 asociado a una asignatura del Grado en Comunicación Audiovisual impartido en la Universitat Politécnica de Valencia- Campus de Gandia, partiendo de los buenos resultados obtenidos en una experiencia piloto el curso anterior. El objetivo es mejorar la comunicación entre alumnado y profesorado, aumentar y mejorar la atención en la asignatura fuera del tiempo de docencia en el aula y, paralelamente, mostrar un uso responsable del móvil y las redes sociales, que pueden tener una utilidad docente. Para ello, se emplea una metodología cuantitativa y cualitativa, basada en formularios y valoración de resultados, así como en la revisión de otros proyectos de uso de redes sociales en el ámbito universitario y bibliografia al respecto.

Palabras clave: Transmedia; Redes sociales; Instagram; Docencia universitaria; Generación Z; Internet

\section{Abstract}

In the actual media environment, technological advances have resulted in new forms of communication based on the Internet, smart devices, and social networks. In this context, universities receive students belonging to the so-called generation $Z$, young people who do not know a world without the Internet, which their smartphone 
regularly accompanies and who use social networks as the primary communication tool, mainly Instagram. Since this generation is based on these practices related to technology and the development of transmedia skills (Scolari, 2018), they can be used in the university classroom to facilitate teaching by integrating them into the teaching-learning processes.

This research aims to put into practice the implementation of the transmedia skills of young people in university education with the case of the creation in the 2019-20 course of an Instagram channel associated with a subject of the Degree in Audiovisual Communication taught at the Universitat Politécnica de València- Gandia Campus, based on the good results obtained in a pilot experience the previous year. The objective is to improve communication between students and teachers. Also, to increase and enhance the attention in the subject outside the teaching time in the classroom and, in parallel, show responsible use of mobile and social networks, which can have a teaching utility. For this, we use a quantitative and qualitative methodology based on the forms and evaluation of the results as well as, in the review of other projects of social networks use in the university field and the bibliography regarding this.

Key Words: Transmedia; Social networks; Instagram; University teaching; Generation Z; Internet

\section{Resumo}

No ambiente de mídia atual, os avanços tecnológicos resultaram em novas formas de comunicação baseadas na Internet, dispositivos inteligentes e redes sociais. Nesse contexto, as universidades recebem estudantes pertencentes à chamada geração $Z$, jovens que não conhecem um mundo sem Internet, acompanhado constantemente pelo smartphone e que utilizam as redes sociais como principal ferramenta de comunicação, principalmente o Instagram. Levando em conta que a vida desta geração é baseada nessas práticas relacionadas à tecnologia e ao desenvolvimento de habilidades transmídia (Scolari, 2018), elas podem ser usadas na sala de aula da universidade para facilitar o ensino, integrando-as aos processos de ensino-aprendizagem. .

Na presente investigação, estuda-se a implementação de competências transmídia no ensino universitário, especificamente no caso de um canal do Instagram 


\section{MONOGRÁFICO}

criado no ano acadêmico de 2019-20 associado a uma disciplina da Licenciatura em Comunicação Audiovisual ministrada na Universitat Politécnica de Valencia - Campus Gandia, com base nos bons resultados obtidos em uma experiência piloto no ano anterior. 0 objetivo é melhorar a comunicação entre alunos e professores, aumentar e melhorar a atenção na disciplina fora do tempo de ensino em sala de aula e, paralelamente, mostrar um uso responsável das redes móveis e sociais, que podem ter uma utilidade de ensino. Para isso, utiliza-se uma metodologia quantitativa e qualitativa, baseada em formas e avaliação de resultados, bem como na revisão de outros projetos de uso de redes sociais no campo universitário e bibliografia a esse respeito.

Palavras chave: Transmedia; Redes sociais; Instagram; Ensino universitário; Geração Z; Internet

\section{Introducción}

Resulta innegable que para los jóvenes universitarios los dispositivos móviles y las redes sociales forman parte de su vida. Estos jóvenes pertenecen a la conocida como Generación Z, nacidos entre 1994 y 2010 (Vilanova y Ortega, 2017), en un momento de expansión masiva de Internet. Esta Generación $\mathrm{Z}$ domina la tecnología y ha crecido en un momento de pleno auge de las redes sociales (Fumero, 2016; Montaña, 2018; Álvarez, Heredia y Romero, 2019). Las nuevas herramientas tecnológicas, como son las mismas redes sociales, pero también los blogs y plataformas de vídeo como YouTube, les han dado el poder de compartir, crear, informar y comunicarse, convirtiéndose todo ello en un elemento esencial en sus vidas (Gómez, Roses y Farías, 2012; Del Barrio y Ruiz, 2014; Cerezo, 2016). Son diversos los estudios que muestran esa estrecha relación de los jóvenes con las tecnologías, concretamente con el smartphone, que los acompaña constantemente. En este sentido, señalamos el estudio del Pew Research Center (2018) que sitúa en un 95\% el porcentaje de los jóvenes entre 14 y 24 años que tiene un smartphone, aumentando en un $22 \%$ en los últimos tres años. En España, el estudio realizado por el Centro Reina Sofía y la Fundación Mapfre sobre jóvenes en el mundo virtual (2018) emplaza al smartphone como el dispositivo del que disponen de forma mayoritaria los jóvenes y que más utilizan para conectarse a Internet tanto en el hogar como en movilidad. Según este mismo informe, la mayoría de estos jóvenes -más del 91\%- 
tiene un perfil en redes sociales que utiliza habitualmente. A todo ello, añadimos que los jóvenes entre 16 y 24 años son los que más se conectan a Internet (ONTSI, 2018), por delante del resto de franjas de edad. Teniendo en cuenta estos datos, no resulta extraño que sea el smartphone el dispositivo más empleado también para el acceso a redes sociales. Esta es precisamente la tercera actividad más habitual que se realiza a través de Internet, solo por detrás de la consulta de servicios de mensajería instantánea, concretamente de WhatsApp, y de la consulta del correo electrónico (Fundación Telefónica, 2019).

Entre las redes sociales, es Instagram en la que principalmente se mueven los jóvenes entre 16 y 30 años, situándose el 70\% de sus usuarios en esta franja de edad (IAB, 2019) por lo que se puede afirmar que es ya un medio social central en la vida de los adolescentes (Márquez y Lanzeni, 2018). De hecho, como señala Marcelino (2015), en los últimos años se produce una migración de los jóvenes de Facebook a Instagram auspiciada por diversos factores como la facilidad para el contenido visual y sencillo, la presencia en la misma de sus ídolos juveniles y marcas de referencia y la percepción que les otorga una mayor libertad en comparación con otras redes similares. En este sentido, se hace referencia a un público más adulto en Facebook, que coincidiría con el crecimiento en Instagram del público juvenil. Efectivamente, Facebook adquirió Instagram en 2012 por lo que se podría pensar en la diferenciación de dos tipos de target para cada red social. Por lo tanto, se puede afirmar que Instagram es el servicio de red social más popular entre los jóvenes, que utilizan esta plataforma durante el tiempo académico y el tiempo de ocio, formando parte de su vida cotidiana y como elemento central en las interacciones sociales (Kofoed y Larsen, 2016; Márquez y Lanzeni, 2018). En esta interacción es destacable la preeminencia del contenido audiovisual a través de los Stories, o historias de Instagram, que ocupan un lugar destacado en el perfil. Este contenido se caracteriza por su brevedad, al tener una duración máxima de 15 segundos, y por ser efímero, ya que solamente aparecerá durante 24 horas tras ser publicado. Para vídeos de mayor duración, la red social dispone de la herramienta InstagramTV.

De esta manera, y como se ha visto en líneas anteriores, en el nuevo ecosistema mediático y comunicativo en el que nos encontramos basado en las aplicaciones, 


\section{MONOGRÁFICO}

las redes sociales y las nuevas plataformas de contenido audiovisual, los jóvenes conviven con las pantallas de un modo espontáneo y natural (Marcelino, 2015; Alonso, 2017; Terol, 2018). En este contexto, cobra una especial relevancia el aprovechar las competencias transmedia de los jóvenes para su aplicación al ámbito educativo.

“Competencias como la navegación web, la acumulación de información, hacer y compartir fotos, coordinar diferentes niveles de comunicación, construir una identidad en un entorno virtual, mirar una serie online, o gestionar la privacidad y la identidad personal en las plataformas online son las mínimas competencias básicas para navegar por un entorno digital". (Scolari, 2018).

Si a principios del siglo XXI hablábamos de alfabetización mediática para "abordar todos los aspectos, objetivos, contenidos, implicaciones, etc. relacionados con la presencia e importancia de los medios en nuestra sociedad" (Gutiérrez y Tyner, 2012), en el actual contexto comunicativo "el alfabetismo transmedia sitúa las redes digitales y las experiencias con los medios interactivos en el centro de su experiencia práctica y analítica" (Scolari, 2018). Se trata de aplicar dentro del entorno educativo los conocimientos y competencias transmedia de los jóvenes, teniendo en cuenta que se cumplan las dos condiciones de las narrativas transmedia: la expansión del relato por diferentes medios y plataformas y la participación activa de los usuarios en ese proceso de expansión (Jenkins, 2003, 2006, 2013; Scolari, $2009,2013,2019)$ con lo que las posibilidades de participación e implicación de los estudiantes se amplían a partir del diseño del aprendizaje situado, significativo y basado en la actividad de los alumnos (Molas, 2018: 59). En este sentido, las redes sociales juegan un papel fundamental porque permiten crear contenido, compartirlo e interactuar con él, pero, además, los jóvenes crean sus identidades según lo que ven en las redes, las cuales son las principales fuentes de información que emplean en cuanto a tendencias y noticias (Marinas, 2019: 188).

Por todo ello, resulta interesante pensar en esas capacidades transmedia de los jóvenes junto con las ventajas de las redes sociales y proponer nuevas fórmulas para sacarles partido por parte del profesorado en la enseñanza universitaria. 
En este sentido, se ha comprobado que las redes sociales son las herramientas de la red en las que los alumnos se mueven con más facilidad, por lo que el reto principal consiste en aprovechar ese entorno para facilitar la docencia integrándolas en los procesos de enseñanza-aprendizaje. Esto "puede ayudar a mejorar la comunicación en el proceso docente, estimular la atención de los estudiantes, promover aprendizajes significativos y optimizar el esfuerzo de todos los participantes en el proceso" (Cabrera et ál., 2013).

Diversos estudios abordan el modo en que se puede enriquecer el proceso de enseñanza-aprendizaje con las redes sociales. Una interesante revisión al respecto la realizan Hortigüela y Pérez (2015) a partir de la cuestión de cómo conseguir que las redes sociales se utilicen en el aula con un criterio racional y un carácter educativo, poniendo el foco en su utilidad como medio para la difusión y profundización de los contenidos abordados en clase y no en la enseñanza de su uso, para conseguir aumentar la motivación y el interés del alumnado.

En esta línea, se pueden aprovechar las ventajas que ofrecen las redes sociales y las mencionadas competencias transmedia de los estudiantes para sacarles partido por parte del profesorado $\mathrm{y}$, a su vez, mostrarles que pueden ser una herramienta útil para su formación académica. Para ello, es fundamental la implicación del profesorado que también han de poner en juego sus propias competencias transmedia y utilizar las redes sociales como medio de comunicación con el alumnado.

“Los profesores pueden fortalecer su credibilidad entre sus estudiantes manifestando y comprendiendo la cultura contemporánea de sus alumnos. [...] Siguiendo estas estrategias podemos convenir que los alumnos pueden percibir el uso que sus profesores hacen de las redes como un intento de fomentar relaciones positivas con ellos, lo cual tendrá una repercusión también positiva en los resultados del alumnado" (Amici y Taddeo, 2018).

De hecho, en estudios como el de Gómez, Roses y Farías (2012), el de Cabrera et ál. (2013) o el de Santoveña y Bernal (2019) sobre el uso académico de las redes sociales, se pone de manifiesto la consideración positiva de las redes sociales por parte de los estudiantes como recurso docente y desde el punto de vista educativo 
como un elemento motivador importante, que ayuda a establecer lazos en el grupo y a mejorar el clima del aula y de la asignatura. A todo ello, se añade el desarrollo de competencias con las redes sociales que marca el Espacio Europeo de Educación Superior, para corroborar la relevancia de las mismas en el proceso enseñanzaaprendizaje en el ámbito universitario.

Por tanto, teniendo en cuenta y conjugando los factores mencionados como son la estrecha relación de los jóvenes universitarios con las tecnologías, una educación transmedia que tenga en cuenta las competencias digitales de estos jóvenes de la Generación Z en la etapa universitaria en la que se encuentran, la consideración positiva entre el alumnado del uso académico de las redes sociales y el auge de la red social Instagram en este grupo de edad, se pueden diseñar estas acciones destinadas a adaptar el proceso de enseñanza-aprendizaje al nuevo entorno y conseguir así una mayor implicación del alumnado, optimizar la comunicación con el profesorado y obtener mejores resultados.

En este sentido, se han llevado a cabo diversas experiencias docentes en el uso de redes sociales en asignaturas de Grado. En referencia concretamente a la red social Instagram, objeto del presente estudio, se pone el caso de la asignatura Proyectos 1 en la Escuela Técnica Superior de Arquitectura y Edificación de la Universidad Politécnica de Cartagena en la que la introducción de Instagram contribuyó a "mantener la atención a la asignatura durante un tiempo extra que no se cernía al horario lectivo docente" (Moreno, 2018). Asimismo, se encuentran otras experiencias en el uso docente de Instagram en asignaturas del Grado de Periodismo en la Universidad CEU San Pablo y de la Universidad de Barcelona que, en el curso 2018/2019 y 2019/2020 respectivamente, han creado un canal de Instagram asociado a la asignatura en el que comparten material didáctico y prácticas de aula entre otros, a través de publicaciones tanto en el feed como en stories con un buen resultado en cuanto a seguimiento y participación del alumnado. Se destaca también la experiencia llevada a cabo por los autores de la presente investigación durante el curso 2018/2019 en la asignatura Teoría y Estética de la Comunicación de Masas, que se imparte en el primer curso del Grado en Comunicación Audiovisual del Campus de Gandia de la Universitat Politécnica de Valencia. En este caso, se pusieron en marcha diversas acciones con la finalidad de optimizar la comunicación profesorado-alumnado en la asignatura, con avisos, notificaciones y ampliación 
de contenidos docentes a través de publicaciones y de stories de Instagram. Todo ello con un balance positivo por lo que Instagram significa como apoyo a la docencia a la hora de ampliar contenidos y aproximar al docente a su público objetivo para conseguir que los alumnos realicen un mejor seguimiento de la asignatura.

De esta manera, partiendo de lo dicho anteriormente, se plantea como objetivo implementar las competencias transmedia de los jóvenes en la enseñanza universitaria con la creación de un canal de Instagram asociado a una asignatura del Grado en Comunicación Audiovisual impartido en el Campus de Gandia de la Universitat Politècnica de València. En relación con lo anterior, se plantean los siguientes objetivos específicos:

1. Mejorar la comunicación entre alumnado y profesorado.

2. Aumentar la atención en la asignatura fuera del tiempo de docencia en el aula.

3. Enseñar un uso responsable del móvil y las redes sociales, que pueden tener una utilidad docente, pero sin ser percibidas por los estudiantes como recurso educativo.

4. Comprobar si se produce un mejor aprovechamiento de la asignatura por parte del alumnado.

5. Mejorar el conocimiento del alumnado y confirmar que la red social en la que pasan más tiempo es Instagram.

\section{Metodología}

Para la presente investigación se emplea una metodología cuantitativa y cualitativa, examinando la respuesta del alumnado a la cuenta de Instagram @ procesoradiofonico que se crea asociada a la asignatura Proceso de Realización Radiofónica, de primer curso del Grado en Comunicación Audiovisual impartida en el Campus de Gandia de la Universitat Politécnica de València. Se trata de una asignatura cuatrimestral de carácter obligatorio de 4,5 créditos, que cuenta con 6 unidades didácticas más el proyecto transversal, que es un trabajo común a todas las asignaturas del cuatrimestre cuya metodología es el aprendizaje basado en proyectos (ABP). 
Esto se lleva a cabo cuantificando el número de alumnos presentes y activos en la cuenta de la red social Instagram y las interacciones con las publicaciones, que se realizan a través de los stories. Asimismo, se contrastan los resultados de la implementación en la asignatura con la percepción que los alumnos tienen de la misma.

\subsection{Encuesta inicial y creación del perfil de Instagram de la asignatura}

Con la mencionada finalidad, se realiza una encuesta previa al alumnado matriculado en la asignatura -un total de 86 alumnos, un 53\% mujeres y un $47 \%$ hombres- en la que se les pregunta por su predisposición a emplear la red social Instagram para comunicarse con el profesor y consultar avisos, notificaciones y material docente a través de un perfil asociado a la asignatura en esta red. También se les consulta sobre la red social en la que se desenvuelven mejor. Las cuestiones planteadas y opciones de respuesta las vemos en la tabla 1.

\begin{tabular}{|l|l|}
\hline \multicolumn{1}{|c|}{ Preguntas } & Opciones de respuesta \\
\hline \multirow{2}{*}{ ¿Eres usuario de Instagram? } & Sí \\
\cline { 2 - 2 } & No \\
\hline \multirow{2}{*}{$\begin{array}{l}\text { ¿Considerarías útil un perfil de la asignatura en la red social } \\
\text { notificaciones, avisos y materiales? }\end{array}$} & Sí \\
\cline { 2 - 2 } & No \\
\hline \multirow{2}{*}{ ¿En qué red social te desenvuelves mejor? } & Instagram \\
\cline { 2 - 2 } & Facebook \\
\cline { 2 - 2 } & Twitter \\
\hline
\end{tabular}

Tabla 1: Cuestiones previas. Elaboración propia.

En la tercera sesión de la asignatura se da a conocer al alumnado el perfil @procesoradiofonico y se informa sobre la publicación de stories con contenidos relacionados con la asignatura por parte del profesorado. El hecho de que sean este tipo de publicaciones se debe a comprobar la inmediatez con la que los alumnos consultan el perfil e interactúan con las mismas, ya que tienen una duración de 24 horas. 


\subsection{Encuesta final}

Una vez finalizado el período lectivo se les vuelve a consultar, en esta ocasión mediante otra encuesta sobre su percepción de la implementación de Instagram como recurso docente en la asignatura. En la tabla 2 vemos las preguntas y opciones de respuesta.

\begin{tabular}{|c|c|}
\hline Preguntas & Opciones de respuesta \\
\hline \multirow{2}{*}{ ¿Sigues la cuenta de Instagram de la asignatura? } & Sí \\
\hline & No \\
\hline \multirow{3}{*}{ ¿Has consultado la cuenta durante el período lectivo? } & $\begin{array}{l}\text { Sí, específicamente para con- } \\
\text { sultar la cuenta de la asig- } \\
\text { natura y ver si había alguna } \\
\text { publicación nueva. }\end{array}$ \\
\hline & $\begin{array}{l}\text { Si, pero solo al consultar mi } \\
\text { perfil de Instagram. }\end{array}$ \\
\hline & No \\
\hline \multirow{2}{*}{$\begin{array}{l}\text { ¿Has interactuado con el perfil de la asignatura para resolver } \\
\text { dudas? }\end{array}$} & Sí \\
\hline & No \\
\hline \multirow{2}{*}{$\begin{array}{l}\text { ¿Consideras que ha aumentado tu atención e interés en la asig- } \\
\text { natura al contar con una cuenta de Instagram? }\end{array}$} & Sí \\
\hline & No \\
\hline
\end{tabular}

Tabla 2: Cuestiones posteriores. Elaboración propia.

Con la finalidad de obtener una visión completa sobre la cuestión planteada, se pone el foco en el interés mostrado en Instagram, materializado en el seguimiento de la asignatura en dicha red social, la utilidad que se le ha dado al perfil de la asignatura y la repercusión que la implementación de Instagram como recurso docente ha tenido en la atención y dedicación prestadas a la asignatura.

\subsection{Docencia inversa o Flipped Classroom}

Por otra parte, en la asignatura objeto de estudio se implementa la metodología de la docencia inversa, o Flipped Clasroom, que posibilita una interacción más personalizada entre docentes y alumnado mediante la utilización de medios tecnológicos y redes sociales que promuevan el trabajo autónomo del alumnado (Lage 
et ál., 2000; Bergmann y Sams, 2012; Roach, 2014; López et ál., 2015; Carabal et ál., 2018).

\section{Resultados}

A continuación, se detallan en primer lugar los resultados de la encuesta previa al inicio del curso, en donde el objetivo es saber si lo que se va a llevar a cabo tiene o no sentido. Luego se explican con detenimiento las diferentes publicaciones que desde la asignatura se han compartido por parte del profesorado y las interacciones del alumnado. Estas publicaciones se agrupan atendiendo a su finalidad. Por último, se exponen los resultados de la encuesta pasada al alumnado al finalizar la asignatura, en la que trasladan su opinión, y si se han seguido todas aquellas recomendaciones y mensajes que se les han mandado a través de la red social Instagram.

\subsection{Encuesta inicial}

Al inicio del cuatrimestre se les pide a los alumnos que están matriculados en la asignatura que rellenen una breve encuesta online. En primer lugar, se les pregunta por su año de nacimiento, con el fin de poderlo relacionar con aquellas características de la Generación $\mathrm{Z}$ que se han comentado anteriormente. El resultado es que 80 de los 86 alumnos han nacido entre los años 1999 y 2001, mientras que los otros 6 han nacido antes de 1994, por lo que sus respuestas quedarían fuera del presente estudio.

A la pregunta si son usuarios de Instagram y tienen creado su perfil en esta red social, la respuesta de los 80 alumnos es un sí; todos se encuentran, con mayor o menor actividad, presentes en Instagram. Se les pregunta, a su vez, si considerarían útil seguir al perfil de la asignatura en esta red social para poder mantener una comunicación más directa con el profesorado y recibir información acerca de la asignatura. Ante esta pregunta, 28 personas responden negativamente, un 35\% del total, mientras que el otro $65 \%$ lo hace de manera afirmativa, señalando que les parece positivo que se cree este vínculo entre el alumnado y la asignatura. 
Respecto a la red social en la que mejor se desenvuelve el alumnado, Instagram es la que obtiene un mayor porcentaje de respuestas con el $46 \%$ y Twitter queda en un segundo lugar con el $18 \%$ de las respuestas. Por otra parte, Facebook cuenta con un $10 \%$ de la clase con perfil y acceso con mayor o menor asiduidad, descartando la posibilidad de poder comunicarse con ellos mediante esta vía.

\subsection{La utilización de Instagram en la asignatura Proceso de Realización Radiofónica}

Durante el curso se publicaron diversas historias en el perfil de Instagram de la asignatura. Una gran mayoría de las mismas fueron imágenes fijas con algún gif animado, con el objetivo de lograr una mayor interacción con los estudiantes. De esta manera, se pueden clasificar las publicaciones en: canal de información, libros recomendados, enlaces a material adicional, recordatorios para la siguiente sesión o prácticas de laboratorio.

\section{Canal de información}

En este apartado se encuentran los anuncios, notificaciones y recordatorios de fechas importantes. Este contenido se notifica de manera oficial vía correo electrónico de la universidad y está disponible en la plataforma institucional, pero también se publica a través de las historias de la red social Instagram con el fin de llegar al alumnado mucho más rápido. Son cursos, jornadas o congresos que se han celebrado en el Campus relacionados con la titulación de Comunicación Audiovisual (GCAV) los que se han difundido por este medio.

Con la publicación del cartel de una jornada sobre el papel de la mujer en los medios de comunicación, que aparece en la figura 1, como una historia de Instagram en la cuenta de la asignatura, este tuvo un total de 148 impresiones, se compartió una vez, dos personas visitaron el perfil de la asignatura y otras diez accedieron al perfil del campus universitario que estaba etiquetado en la publicación, según los datos que la propia red social ofrece en su aplicación para smartphone. 


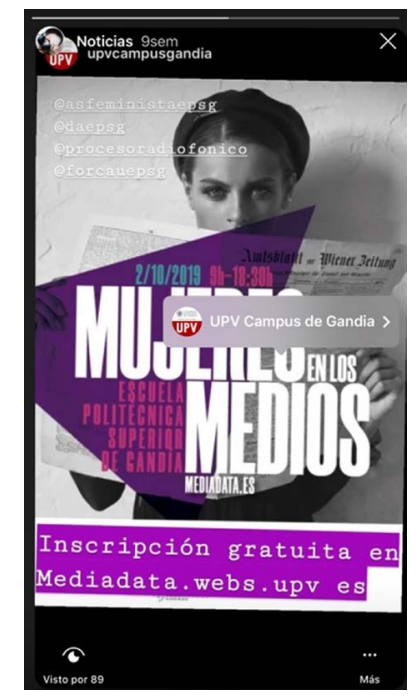

Figura 1: Storie informativo publicado por la cuenta @procesoradiofonico (Elaboración propia).

En el presente caso de estudio se publicaron diversas historias relacionadas con los fenómenos climatológicos adversos. En concreto, referidas a la suspensión 0 no de las clases y su reanudación tras sufrir en estos dos últimos años distintos episodios de la gota fría, o lo que técnicamente se conoce como DANA (Depresión Aislada en niveles altos). En los meses de octubre y noviembre de 2018 y en septiembre de 2019 exactamente fue cuando urgió avisar a los alumnos de manera rápida para informarles que, o bien se suspendían las clases ante la alerta que había dado la Agencia Estatal de Meteorología (AEMET), o se reanudaban tras volver a la normalidad y cancelarse la alerta por parte de la propia AEMET.

Además, se empleó el recurso de las historias de Instagram para dar información al alumnado de que ya se encontraban disponibles en la plataforma de la universidad, así como en su correo electrónico, las calificaciones de ejercicios o pruebas escritas evaluables. 


\section{Libros recomendados}

La guía docente de la asignatura define, además de otorgarle un porcentaje de la nota final, la realización de un trabajo sobre un libro cuya temática se encuentre ligada a la asignatura. Para ello, se les facilitó a los alumnos un listado de más de un centenar de libros para que cada estudiante escogiese uno y así compartir con los compañeros el trabajo sobre la lectura que han realizado y sus consideraciones sobre dicha lectura. Al margen del listado, se publicaron algunas historias en Instagram informando al alumnado de que disponen de una biblioteca en el Campus de Gandia donde poder buscar los ejemplares disponibles y elegir aquel que les parezca más atractivo para su lectura. Asimismo, se ofrecieron varias historias con novedades editoriales y publicaciones que acaba de adquirir la biblioteca recientemente.

\section{Enlaces a material adicional}

Entre estas publicaciones se encuentran todas aquellas que incorporaron a la historia de Instagram un enlace para ampliar información con material audiovisual de refuerzo. No se consideró necesario que los vídeos fueran de producción propia, se compartieron vídeos de otras cuentas con información relevante y aportación de contenido al temario de la asignatura. Un ejemplo en el presente caso de estudio es uno de los vídeos que la Cadena Ser publica en InstagramTV. Se trata de un reportaje radiofónico al que se ha incorporado una imagen fija y en el que el periodista de la Cadena SER Severino Donate realiza un recorrido por la dilatada trayectoria de la radio como medio de comunicación.

\section{Recordatorios para la siguiente sesión}

La asignatura Proceso de Realización Radiofónica, sobre la que se basa el presente caso de estudio, está inmersa en el programa de Docencia Inversa que lleva a cabo el Vicerrectorado de Recursos Digitales y Documentación de la Universitat Politècnica de Valencia. En este sentido, se realizaron publicaciones en las que se informa de toda la documentación que debían leerse para la siguiente sesión, las actividades a realizar tanto fuera como dentro del aula o los ejercicios para entregar. De esta manera, el alumnado se encuentra imbricado con el desarrollo del llamado Flipped Classroom, para así llegar al aula con las tareas ya realizadas. 


\section{MONOGRÁFICO}

\section{Interacción prácticas de laboratorio}

Esta tipología de historias de Instagram es la que está relacionada con compartir todas aquellas imágenes y vídeos que los alumnos han publicado en sus cuentas personales, con el fin de que el resto de la clase y aquellos que no han podido asistir sean conocedores de las actividades que se han desarrollado en la sesión de prácticas de laboratorio. El objetivo de este tipo de publicaciones es motivar al alumno en torno a la asignatura y a los contenidos de la misma, girando sobre su actividad práctica en las sesiones de laboratorio llevadas a cabo en el aula de informática 0 , en la mayoría de las ocasiones, en el estudio de radio y las cabinas de grabación.

\subsection{Encuesta posterior}

Una vez finalizadas las clases de la asignatura Proceso de Realización Radiofónica y realizada la prueba de evaluación, se les pidió a los alumnos que realizaran una nueva encuesta mediante la que poder disponer de los datos necesarios para seguir adelante con la presente investigación.

En esta ocasión, se acudió a los alumnos que cursaban la asignatura nacidos después de 1994, con el fin de discernir algunas dudas tras la puesta en práctica del perfil de la asignatura en Instagram y la publicación de diferentes historias a lo largo del curso. Se les planteó si consultaron la cuenta de Instagram durante

el período lectivo, a lo que el $71 \%$ de los alumnos respondió que sí la consultaron al entrar a consultar su propio perfil, mientras que el $28 \%$ entraron a Instagram específicamente para consultar el perfil de la asignatura y solo un 1\% no entró en ningún momento. La siguiente cuestión consistió en conocer si habían interactuado con el perfil de la asignatura o con el del profesor para resolver cualquier tipo de duda que les hubiese surgido. A esta pregunta 32 alumnos contestaron afirmativamente, un $40 \%$ del total, mientras que los 48 restantes señalaron no haber interactuado ni una sola vez con el profesor. De ese $40 \%$, diez personas marcaron la opción que fijaba entre 1 y 4 las veces que habían escrito algún mensaje al profesor, 18 alumnos los que interactuaron entre 5 y 10 veces y solo cuatro personas las que consultaron al profesor en más de 10 ocasiones. 
Finalmente, se les planteó si consideraban que el hecho de estar presentes en Instagram había aumentado el interés por la materia impartida durante el curso. Un 90\% respondió de manera afirmativa, 72 de los 80 alumnos consideraron que sí les había potenciado el interés por la asignatura en cuestión, superando las expectativas que tenían prefijadas al inicio de curso. Solo 8 personas respondieron que no les había influido en absoluto el hecho de disponer de perfil en Instagram en su percepción de la asignatura.

\section{Discusión}

La experiencia analizada en el presente caso de estudio, el de la asignatura Proceso de Realización Radiofónica de primer curso del Grado de Comunicación Audiovisual, resulta completamente positiva en relación con el uso de la red social Instagram por parte de los alumnos nacidos después de 1994, como se desprende del análisis de los resultados. Se comprueba el uso mayoritario de la red Instagram por parte del alumnado, en la que la mayoría disponen de un perfil, en detrimento de otras redes sociales, como Twitter y Facebook, cuyo uso es minoritario. Asimismo, se aprecia con el estudio que las historias, o stories, de Instagram pueden convertirse en una herramienta muy eficaz que cumpla los objetivos propuestos, en concreto conseguir un mayor aprovechamiento de la asignatura por parte del alumnado y aumentar la atención de la asignatura fuera del tiempo docente en el aula.

Por otra parte, existe un interés específico entre el alumnado por el perfil de la asignatura, al constatarse un porcentaje que accedió a la red social solo para consultar sus publicaciones. Además, la mayor parte del alumnado que acudió a la red social para ver su propio perfil también consultó el perfil de la asignatura, lo que indica que la práctica totalidad del alumnado consultó el perfil de la asignatura Proceso de Realización Radiofónica a lo largo del período lectivo.

Por tanto, se puede afirmar que la utilización de esta red social como canal para que el docente y su alumnado se comuniquen va a establecer unas relaciones más cercanas y a fomentar el interés hacia la asignatura y a despertar inquietudes entre sus alumnos. 
En este sentido, se propone una clasificación para tratar de ayudar a todos aquellos docentes que decidan aplicar esta manera para conectar con sus alumnos. Todas las diferentes historias que se han publicado a lo largo del curso quedan clasificadas entre las diferentes tipologías de canal de información, libros recomendados, enlaces a material adicional, recordatorios para la siguiente sesión y la interacción con el alumnado ante prácticas o ejercicios.

Resulta de interés la siguiente consideración: la publicación de estos contenidos da respuesta también al objetivo de enseñar un uso responsable y proporcionar una utilidad docente tanto al teléfono móvil como a las redes sociales, empleadas por los estudiantes como recurso educativo al acceder a material relacionado con la asignatura a través de Instagram.

Como limitación en la investigación, se señala que el estudio se ha llevado a cabo en una asignatura en la que los alumnos han de pasar unas pruebas evaluativas para superarla. Debido a esta circunstancia, las respuestas ante las encuestas realizadas pueden haberse visto inducidas a resultados positivos.

Teniendo en cuenta lo anterior, resulta conveniente señalar que se trata de un trabajo de investigación que empieza con la implementación gradual en diversas asignaturas. De esta manera, cabe destacar que esta investigación es la continuación de unos estudios previos en los que se analizó en promociones y asignaturas diferentes cómo el profesor, desde su cuenta particular, se comunicaba con los alumnos utilizando Instagram para ello, ya fuese mediante mensajes directos o a través de las historias, aunque sin reemplazar a los pertinentes envíos de los correos electrónicos corporativos, los anuncios en la página web de la universidad y en las diferentes redes sociales de la propia universidad y sus centros.

En este caso, a partir de encuestas previas al alumnado, se comprobó su uso mayoritario de la red social Instagram en la que la gran mayoría disponen de un perfil y el uso más esporádico del correo institucional de la universidad. También la opinión positiva respecto a que el profesorado cuente con un perfil en redes sociales y pueda emplearlo en la comunicación con los alumnos. Estos buenos resultados obtenidos son la base para la implantación de Instagram como herramienta docente 
en el curso 2019/2020, pero, en esta ocasión, como se ha mencionado, creando un canal asociado a la asignatura. Se vuelve a optar por esta red social porque, como hemos visto, es la red que más emplean los jóvenes, junto con YouTube, y por la buena experiencia del curso anterior.

Respecto a esta experiencia anterior, en el presente estudio se plantea como novedad la creación de un perfil propio de la asignatura que los alumnos pueden seguir, de tal manera que se evita que el mensaje llegue a destinatarios no deseados, como lo pueden ser familiares y amigos, centrándose exclusivamente en el alumnado que está matriculado en la asignatura, tras aprobar las solicitudes de seguimiento.

Para concluir, resulta evidente que la sociedad actual avanza tecnológicamente a pasos de gigante. En los últimos años la elevada penetración del smartphone y la evolución que se ha producido en la manera en la que nos comunicamos, ha hecho posible que nos replanteemos si el mensaje del profesor llega a su alumnado de manera óptima y eficaz. Para lograrlo es necesario, como se ha comprobado, que se produzcan una serie de cambios en el aula y que el profesor sea capaz de adaptarse a un público que está acostumbrado al consumo audiovisual y a comunicarse a través de la pantalla. Una necesaria alfabetización transmedia que puede resultar trascendental en el proceso de enseñanza-aprendizaje.

La implantación en muchas universidades de la metodología de la Flipped Classroom implica que se utilicen redes sociales y medios tecnológicos para poder llevar un seguimiento del alumno tanto dentro como fuera del aula. Los alumnos consumen mucho tiempo de su día a día en las redes y tienen gran presencia en ellas. A través del caso estudiado, se considera que puede incrementar su interés hacia la materia impartida y favorecer que el alumno se comunique de manera más directa con su profesor.

De hecho, con todos los aspectos positivos enumerados, la dirección del título de GCAV ha considerado esta vía de mucha importancia para la difusión de las actividades, creando este curso 2019/2020 una cuenta de Instagram para dar a conocer todas aquellas informaciones que sean relevantes para el alumnado, así como compartir los proyectos en los que se encuentran trabajando los alumnos, tanto a nivel académico como personal. 


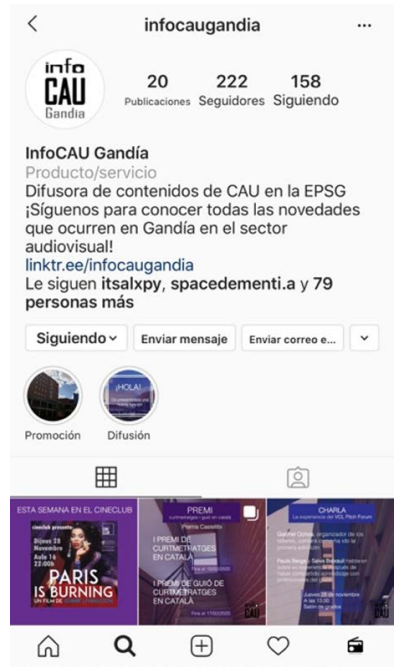

Figura 2: Perfil de la cuenta @infocaugandia (Elaboración propia).

Por lo tanto, se constata que la implementación de Instagram en la enseñanza universitaria puede ser un apoyo a la docencia a la hora de ampliar contenidos y aproximar al docente a su público objetivo para conseguir que los alumnos realicen un mejor seguimiento de la asignatura, lo cual se ve reflejado en mejores resultados en la evaluación, produciéndose, tras la experiencia docente, un mayor aprovechamiento por parte del alumnado de la asignatura y una mejora en la comunicación con el profesorado, lo que abre la puerta a continuar los estudios en esta línea.

\section{Referencias}

Alonso, N. (2017). La reformulación de los medios ante la convergencia digital. En Flores-Vivar, J. M. (coord.). Periodismo en nuevos formatos ( $1^{\text {a }}$ ed., pp. 125139). Madrid: Fragua.

Álvarez, E., Heredia, H. y Romero, M.F. (2019). La Generación Z y las Redes Sociales. Una visión desde los adolescentes en España. Revista Espacio, 20(40). Disponible en http://bit.ly/2VyjG2z

Amici, S., Taddeo, G. (2018). Aprovechando en el aula las competencias transmedia. Un programa de acción. En Scolari, C. A. (ed.). Adolescentes, medios de 
comunicación y culturas colaborativas. Aprovechando las competencias transmedia de los jóvenes en el aula, ( $1^{\mathrm{a}}$ ed., pp.125-135). Barcelona: Universitat Pompeu Fabra.

Bergmann, J. y Sams, A. (2012). Flip your classroom: Reach every student in every class every day, International Society for Technology in Education. New York: International Society for Technology in Education.

Cabrera, M., Díez, R., Teruel, D., Rebollo, M., Poza-Luján, J.L., Calduch-Losa, Á. y Albors, A. (2013). Experiencias docentes en el uso de grupos de Facebook en el aula. II Congreso Internacional sobre Aprendizaje, Innovación y Competitividad (CINAIC 2013). Fundación General de la Universidad Politécnica de Madrid.

Carabal-Montagud, M., Santamarina, V., López, M. y De-Miguel-Molina, M. (2018). Flipped Teaching y Redes Sociales. En IN-RED 2018. IV Congreso Nacional de Innovación Educativa y Docencia en Red, 285-298. Valencia: Editorial Universitat Politècnica de València. https://doi.org/10.4995/INRED2018.2018.8739

Centro Reina Sofía, Fundación Mapfre (2018). Jóvenes en el mundo virtual: usos, prácticas y riesgos. Disponible en: http://bit.ly/2L476C3

Cerezo, P. (2016). La Generación Z y la información. Revista de Estudios de Juventud, 114, 95-109. Disponible en http://bit.ly/2PBaC96

Del Barrio, Á. y Ruiz, I. (2014). Los adolescentes y el uso de las Redes Sociales. International Journal of Developmental and Educational Psychology, 3(1), 571-576. Fumero, A. (2016). Jóvenes y Generación Z. Disponible en: http://bit.ly/2XZfKqK Fundación Telefónica (2019). Sociedad Digital en España. Disponible en http://bit. ly/2DvpTlF

Gómez, M., Roses, S. y Farías, P. (2012). El uso académico de las redes sociales en universitarios. Comunicar, 38, 131-138. https://doi.org/10.3916/C38-2012$\underline{03-04}$

Gutiérrez Martin, A. y Tyner, K. (2012). Educación para los medios, alfabetización mediática y competencia digital. Comunicar, 38, 31-39. https://doi. org/10.3916/C38-2012-02-03

Hortigüela Alcalá, D. y Pérez Pueyo, Á. (2015). Uso de las redes sociales como elemento formativo en el aula: Análisis de la motivación del alumnado universitario. Revista ICON014 Revista Científica De Comunicación y Tecnologías Emergentes, 13(2), 95-115. https://doi.org/10.7195/ri14.v13i2.788 
IAB (2019). Estudio anual de redes sociales 2019. Disponible en http://bit. ly/2R2UPS9

Jenkins, H. (2003). Transmedia Storytelling. Moving characters from books to films to video games can make them stronger and more compelling, en MIT Technology Review, Disponible en: http://bit.ly/34LuvQF

- (2006). Convergence culture. La cultura de la convergencia de los medios de comunicación, Barcelona: Paidós.

Jenkins, H., Ford, S. y Green, J. (2013). Spreadable Media: Creating Value and Meaning in a Networked Culture, Nueva York: NYU Press.

Kofoed, J. y Larsen, M. Ch. (2016). A snap of intimacy: Photo-sharing practices among young people on social media, en First Monday, 21(11). https://doi. org/10.5210/fm.v21i11.6905

Marcelino Mercedes, G. (2015). Migración de los jóvenes españoles en redes sociales, de Tuenti a Facebook y de Facebook a Instagram. La segunda migración. Revista ICON014 Revista Científica De Comunicación Y Tecnologías Emergentes, 13(2), 48-72. https://doi.org/10.7195/ri14.v13i2.821

Marinas, L. (2019). Instagram: Donde Millennials, Generación Z, Mcluhan y Bolter se cruzan. CIC. Cuadernos De Información Y Comunicación, 24, 187-201. https:// doi.org/10.5209/ciyc.64641

Márquez, I. y Lanzeni, D. (2018). Instagram, en Scolari, C. A. (ed.). Adolescentes, medios de comunicación y culturas colaborativas. Aprovechando las competencias transmedia de los jóvenes en el aula, ( $1^{\text {a }}$ ed., pp.105-108). Barcelona: Universitat Pompeu Fabra.

Molas, N. (2018). La guerra de los mundos. La narrativa transmedia en educación, Barcelona: UOC.

Montaña, M. (2018). La nueva generación Z: conectada, pragmática y familiar. En COMeIN, 77. Disponible en: http://bit.ly/2TpJ33V

Moreno-Moreno, $\mathrm{M}^{\mathrm{a}}$. P. (2018). Uso docente de la red social Instagram en la asignatura de Proyectos 1. En VI Jornadas sobre Innovación Docente en Arquitectura, 508518. Barcelona: UPC IDP; GILDA; Zaragoza: Servicio de Publicaciones de la Universidad de Zaragoza. https://doi.org/10.5821/jida.2018.5511

ONTSI (2018). Perfil sociodemográfico de los internautas. Observatorio Nacional de las Telecomunicaciones y de la Sociedad de la Información. Disponible en http://bit.ly/33yHLXd 
Pew Research Center (2018). Teens, Social Media \& Technology 2018. Disponible en: https://pewrsr.ch/2R8tQ03

Roach, T. (2014). Student perceptions toward flipped learning: New methods to increase interaction and active learning in economics. En International Review of Economics Education, 17, 74-84.

Santoveña-Casal, S. y Bernal-Bravo, C. (2019). Explorando la influencia del docente: Participación social en Twitter y percepción académica. Comunicar, 58, 75-84. https://doi.org/10.3916/C58-2019-07

Scolari, Carlos A. (2009): Transmedia Storytelling. Implicit consumers, narrative worlds and branding in contemporary media production. En International Journal of Communication, 3, 586-606. Disponible en http://ijoc.org/ojs/ index.php/ijoc/article/ view/477/336

- (2013): Narrativas transmedia. Cuando todos los medios cuentan, Barcelona: Deusto.

_ (ed.) (2018). Adolescentes, medios de comunicación y culturas colaborativas. Aprovechando las competencias transmedia de los jóvenes en el aula, Barcelona: Universitat Pompeu Fabra.

Scolari, C. A., Lugo Rodríguez, N. y Masanet, Ma . J. (2019): Educación Transmedia. De los contenidos generados por los usuarios a los contenidos generados por los estudiantes. En Revista Latina de Comunicación Social, 74, 116-132. https:// doi.org/10.4185/RLCS-2019-1324

Terol, R. (2018). La utilización de las narrativas transmedia en el entorno de la radio municipal, en Galán, E., Rodríguez, A. y Marzal, J. (eds.). Contenidos transmedia para la radiotelevisión de proximidad, pp. 345-358. Pamplona: EUNSA.

Vilanova, N. y Ortega, I. (2017). Generación Z: Todo lo que necesitas saber sobre los jóvenes que han dejado viejos a los millennials. Barcelona: Plataforma Editorial.

\section{Glosario}

Historias o stories de Instagram: contenido situado en la parte superior del perfil de Instagram. Se caracteriza por su brevedad y por estar disponible solo durante 24 horas tras ser publicado. Recientemente se ha incorporado la opción de guardar estas publicaciones en el apartado Historias Destacadas incluido en el perfil. 
161 | Nadia Alonso López y Raúl Terol Bolinches

MONOGRÁFICO

Feed: Por lo que respecta a la red social Instagram, se trata de la imagen inicial que percibe el usuario al acceder a un perfil, sin entrar a las publicaciones ni a las historias. Esta está conformada por fotografía de perfil, imágenes en miniatura e historias destacas, entre otros.

InstagramTV: Herramienta de Instagram que permite crear vídeos propios de hasta 60 minutos de duración y formato vertical.

\section{(c) (i)}

Este obra está bajo una licencia de Creative Commons Reconocimiento 4.0 Internacional. 